\title{
COMPRENDRE
}

\section{Les lasers à cascade quantique : vers la démocratisation ! ${ }^{1}$}

\author{
Mathieu CARRAS \\ mirSense, 8 avenue de la Vauve, \\ centre d'intégration Nanolnnov, \\ 91120 Palaiseau \\ mathieu.carras@mirsense.com
}

Les lasers à cascade quantique ont fait, depuis leur première démonstration en 1994, des progrès considérables, notamment dans le moyen infrarouge (bande spectrale allant de 3 à $12 \mu \mathrm{m})$. Ils peuvent maintenant être considérés comme des composants matures pour les applications en défense, sécurité et métrologie industrielle et environnementale. Ainsi, ces lasers sont utilisés dans de nombreux systèmes commerciaux. Nous parcourons dans cet article les progrès récents des lasers à cascade quantique, et discutons des nouvelles perspectives.

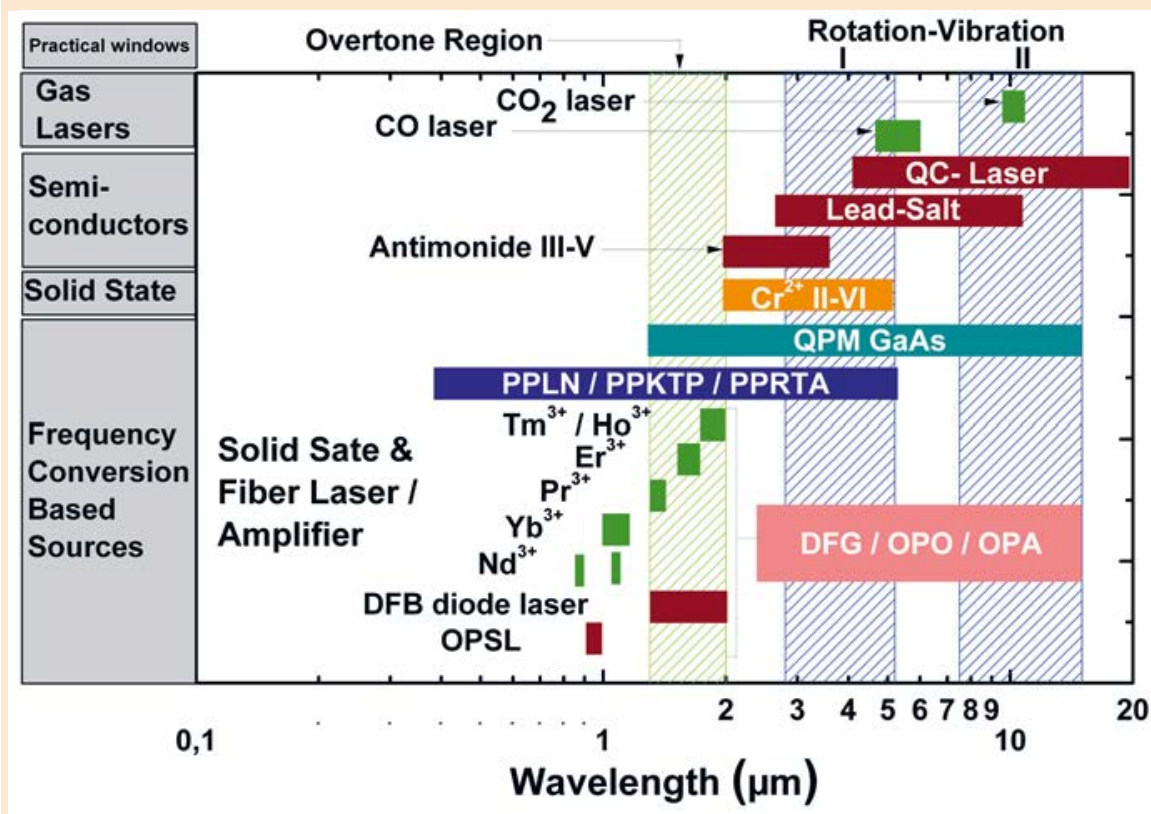

Figure 1. Différentes sources lasers dans le moyen infrarouge utilisées en spectroscopie et leurs plages en longueur d'onde (figure tirée de F. Tittel et al., Topics Applied Physics, 89, 445 (2003), C SpringerNature).

(periodically poled lithium niobate), sont matures, robustes et relativement compactes mais sont limitées à des longueurs d'onde inférieures à $5 \mu \mathrm{m}$ et à de faibles puissances optiques. Les OPOs présentent de fortes puissances crêtes mais sont cependant encore trop volumineux et coûteux pour un 
semi-conducteurs fonctionnant à température ambiante en mode pulsé ou continu dans le moyen infrarouge.

\section{Principe du laser à cascade quantique}

Le QCL est basé sur une transition optique d'un électron entre des niveaux quantifiés de la bande de conduction [2]. Contrairement aux diodes lasers classiques, la longueur d'onde d'émission ne dépend pas directement de la bande interdite des matériaux semi-conducteurs utilisés mais principalement de l'empilement des couches formant la zone active. L'ingénierie quantique et le contrôle de l'épaisseur des couches à la couche atomique près, accessibles par les techniques d'épitaxie de production (figure 2), permettent d'obtenir les niveaux d'énergie et les fonctions d'onde désirés (figure 3). Pour maximiser l'amplification optique, on juxtapose un grand nombre de zones actives, créant ainsi une structure périodique. Sous l'effet d'un champ électrique, les électrons traversent cette structure par effet tunnel puis émettent un photon et sont recyclés d'une période à l'autre. Les électrons décrivent ainsi une cascade d'énergie potentielle en émettant chacun idéalement autant de photons qu'il y a de périodes. La flexibilité unique du dessin des lasers à cascade quantique rend cette technologie à semi-conducteurs très attractive pour la fabrication de lasers sur une large plage spectrale s'étendant jusqu'au térahertz $(\mathrm{THz})$, soit de 2,5 à $160 \mu \mathrm{m}$ [3]. Les meilleures performances sont cependant obtenues dans la bande située entre 4 et $10 \mu \mathrm{m}$. Le système de matériaux utilisés est dans ce cas le même que celui utilisé pour les diodes laser télécom (GaInAs/ AlInAs sur substrat InP).

\section{Applications et performances}

Deux familles de composants QCL peuvent être définies en fonction des applications : les sources de forte puissance, utilisées dans les systèmes

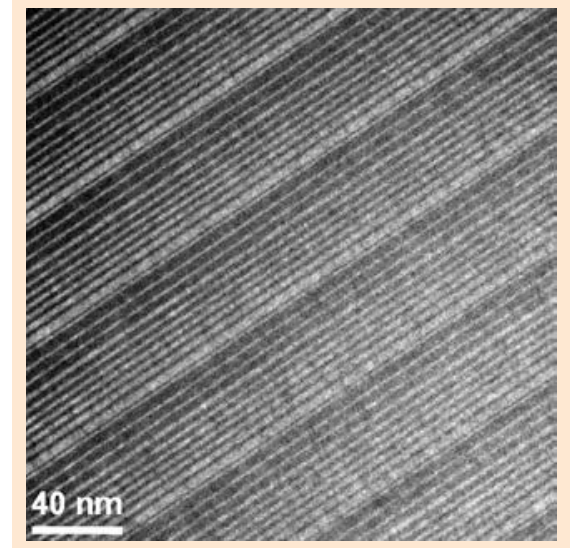

Figure 2. Photographie en microscopie électronique à transmission d'une structure périodique d'un laser à cascade quantique. Les puits quantiques sont en noir et les barrières en blanc.

de contre-mesures optiques pour le brouillage ou l'aveuglement des détecteurs moyen infrarouge; les sources pour la détection des explosifs, des drogues, des agents chimiques de guerre, des toxiques industriels, des agents polluants et le contrôle des procédés industriels. Ces sources pour la spectroscopie peuvent se séparer en deux catégories, les sources mon-fréquences, plus ou moins accordables dans les temps, et les peignes de fréquence, récemment démontrés en utilisant la technologie QCL [4]

Pour ces deux types d'applications, une équipe de l'université de Northwestern a démontré des performances remarquables : une puissance optique de 5 watts est obtenue pour une émission à $4,9 \mu \mathrm{m}$ avec une source multi-mode constituée d'une cavité laser de $5 \mathrm{~mm}$ de long et de $8 \mu \mathrm{m}$ de large. Le laser fonctionne en continu à température ambiante avec un rendement de conversion de $21 \%$, proche de la limite théorique de $30 \%$ [5]. Une source mono-fréquence à $4,8 \mu \mathrm{m}$ avec une puissance optique de 2,4 watts a aussi été démontrée [6]. Ces résultats confirment le fort potentiel des lasers à cascade quantique. Pour les contre-mesures optiques et pour la détection de gaz par spectroscopie, le défi est toujours d'accroître le rendement de conversion électro-optique pour augmenter la puissance optique et/ou réduire la puissance électrique nécessaire au fonctionnement du laser. Au regard
ImegIme Optite

\section{Wavefront sensors and \\ adaptive optics for \\ optical metrology, \\ lasers and microscopy}
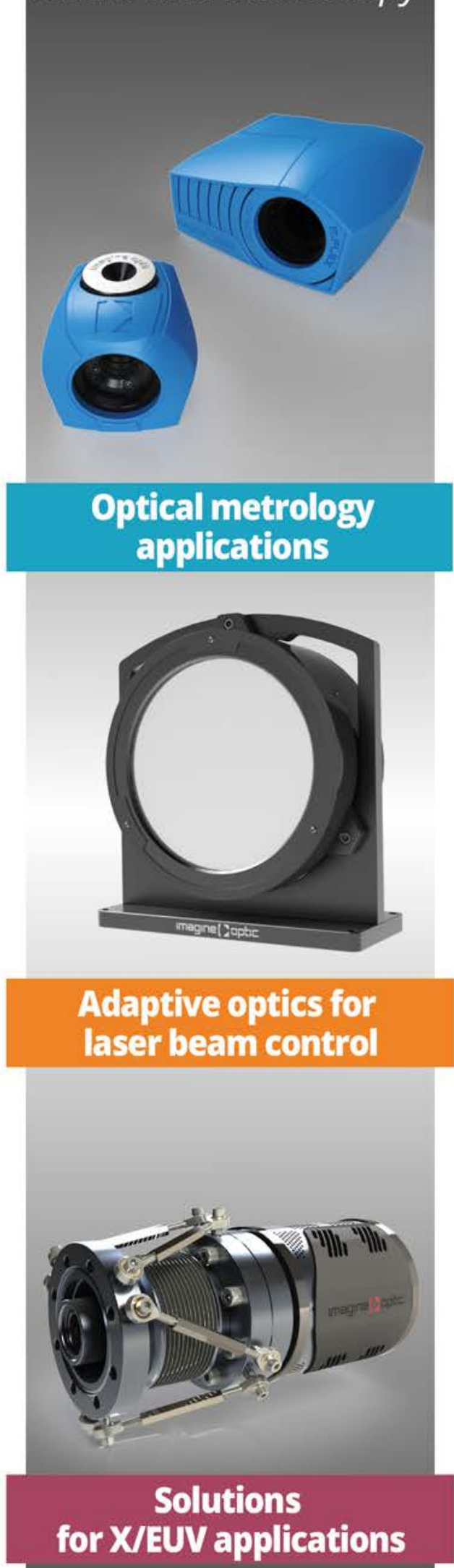

Contact us for more details: contact@imagine-optic.com or +33164861560 


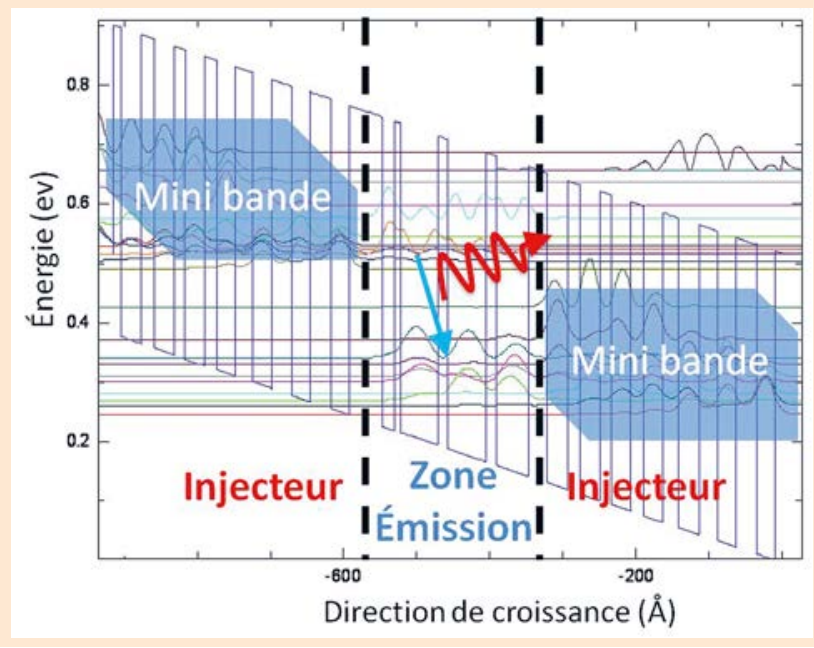

Figure 3. Diagramme typique de la bande de conduction d'un QCL montrant les niveaux d'énergie et les fonctions d'onde sur une période et demie. des performances actuelles, plusieurs équipes imaginent aussi la réalisation de circuits photoniques MIR intégrés pour ajouter de nouvelles fonctionnalités sur la puce semiconductrice tout en restant compacts.

\section{Le très fort enjeu des marchés de masse}

Le besoin de métrologie est omniprésent dans nos vies, avec la concomitance de la dégradation de la qualité de l'air depuis l'ère industrielle et de la prise de conscience de son impact sur la santé des personnes et sur l'environnement. Si les QCLs peuvent remplir ce besoin, il existe aujourd'hui des freins économiques à leur utilisation massive.

Le défi actuel est de faire passer cette technologie de marchés de niche à des applications de volume, et de démocratiser son usage. Les deux verrous actuels sont le coût des lasers et le coût de l'architecture système à mettre en œuvre pour les utiliser. Les performances atteintes permettent d'envisager l'intégration d'architectures photoniques plus complexes pour obtenir de nouvelles fonctionnalités en tirant profit du potentiel considérable des technologies développées autour des semi-conducteurs et de leur maturité et donc de simplifier les architectures système et réduire les coûts. En pratique, cela peut se faire en intégrant ces sources sur des plateformes silicium. C'est aujourd'hui un des axes de développement majeurs de la communauté QCL.

\section{Des sources matures pour la spectroscopie}

La plage spectrale du moyen infrarouge est particulièrement intéressante pour la spectroscopie de gaz car elle présente plusieurs fenêtres de transmission atmosphérique (avec des lignes d'absorption de $\mathrm{H}_{2} \mathrm{O}$ et $\mathrm{CO}_{2}$ faibles et peu nombreuses) où nous trouvons les raies d'absorption ro-vibrationnelles fondamentales de plusieurs polluants atmosphériques ( $\mathrm{NO}, \mathrm{N}_{2} \mathrm{O}, \mathrm{CO}, \mathrm{H}_{2} \mathrm{CO}$ (formaldehyde), $\mathrm{NH}_{3}, \mathrm{SO}_{2}$, etc.). Ces raies fondamentales sont deux à trois ordres de grandeur plus intenses que leurs harmoniques dans le proche infrarouge (1 à 2,5 $\mu \mathrm{m}$ ). De plus, la plupart des molécules organiques complexes (plus de 5 atomes) qui ont un intérêt pour la défense et la sécurité ont aussi leurs signatures entre 7 et $12 \mu \mathrm{m}$.

\section{Fonctionnement mono-fréquence}

Un fonctionnement mono-fréquence du laser est nécessaire pour les applications en spectroscopie. Il est généralement obtenu en introduisant un réseau dans la cavité laser qui induit une contre-réaction répartie (ou DFB, pour distributed feed-back). Ce réseau, qui agit comme un filtre fréquentiel, entraîne ainsi un fonctionnement mono-fréquence. Les difficultés pour dessiner et réaliser un QCL DFB ont été partiellement surmontées en utilisant des réseaux enterrés. Cependant, cette technique nécessite une étape de reprise de croissance onéreuse et critique. Une nouvelle approche basée sur l'utilisation de réseaux métalliques de surface a été développée. Cette nouvelle approche repose sur une modulation de l'indice générée par le couplage entre les modes guidés dans la zone active et un mode confiné à l'interface, entre le métal et le guide supérieur (figure 4) [7]. Précisons que ce couplage est possible car les modes contra-propagatifs d'une part, et le mode de surface d'autre part, ont la même polarisation TM. Ces réseaux métalliques de surface peuvent être utilisés dans le moyen infrarouge car les pertes sont moins élevées que dans le proche infrarouge. Un avantage supplémentaire de cette approche est sa robustesse vis-à-vis des variations technologiques, ce qui permet de contrôler la longueur d'onde d'émission à plus ou moins $1 \mathrm{~cm}^{-1}$. Des QCL DFB fonctionnant en mode pulsé ou continu

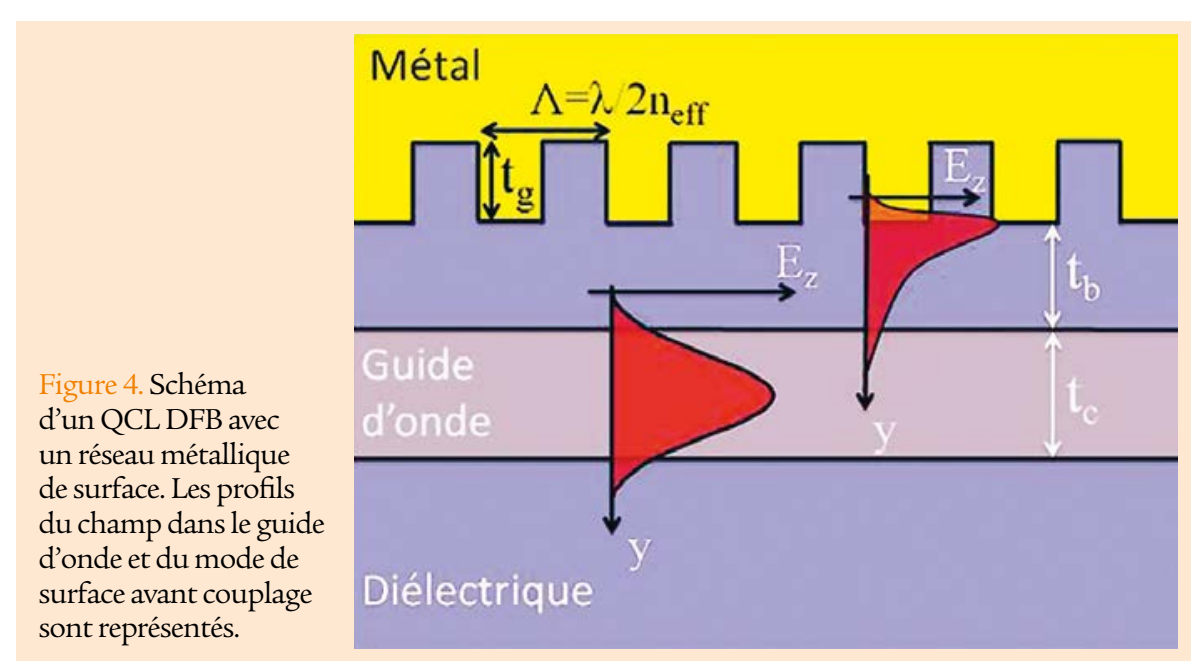


sont disponibles commercialement (voir www.mirsense.com par exemple) sur la totalité de la plage allant de 4 à $10 \mu \mathrm{m}$ [7]. Les lasers fonctionnent à température ambiante sur un module thermoélectrique à effet Peltier. Les puissances émises sont de quelques dizaines de milliwatts et le taux de réjection des modes latéraux (ou SMSR, pour side mode suppression ratio) est supérieur à $30 \mathrm{~dB}$. La longueur d'onde d'émission peut être accordée sur une plage de 3 à $4 \mathrm{~cm}^{-1}$ par échauffement de la structure laser en modulant le courant injecté dans le QCL DFB. Un des avantages cruciaux de cette approche est d'être associable à la fabrication des QCLs sur des chaînes silicium.

\section{La voie vers}

\section{la démocratisation : co-intégration QCL/silicium}

La grande tendance actuelle est l'intégration des matériaux III-V sur silicium, en particulier pour les applications télécoms. Les QCL ne font pas exception et mirSense, en collaboration avec le CEA-LETI, est pionnier dans ce domaine. L'avantage de cette approche est de pouvoir intégrer des fonctions réalisées sur silicium de manière monolithique, engendrant des gains en robustesse, compacité, mais surtout coût et capacité de production avec le volume. Pour cela, il est nécessaire de faire deux choses : ramener les QCL sur le silicium et savoir traiter la lumière émise par les QCls sur le silicium [8].

\section{QCL sur silicium}

Il existe plusieurs solutions pour amener les QCLs sur silicium. La première solution s'appelle l'intégration hétérogène, qui consiste à fabriquer le QCL de manière classique, puis à le reporter sur un substrat de silicium, ou en face d'une puce en silicium. C'est la plus simple, mirSense et le CEA-LETI ont démontré la pertinence de cette approche pour réaliser des sources accordables. L'autre solution consiste à n'avoir que la couche d'épitaxie QCL sur le silicium et à procéder à la fabrication du laser sur le substrat silicium. Là aussi, le CEA-LETI et mirSense ont démontré pour la première fois ce procédé de fabrication sur substrat $200 \mathrm{~mm}$, c'est-à-dire un standard de l'industrie du silicium (travaux à paraître).

\section{Nouveaux matériaux pour guider la lumière sur silicium}

Afin de réaliser des fonctions sur silicium, il a été nécessaire de développer de nouvelles filières de matériau permettant de guider la lumière moyen-infrarouge. En effet, l'approche classique de guidage de la lumière sur silicium, utilisant des guides avec un corps en silicium et des couches guidantes en silice ne fonctionne plus au-delà de $4 \mu \mathrm{m}$ de longueur d'onde. Différentes propositions ont été faites, principalement en introduisant du germanium. La première filière couvrant la bande 3-8 $\mu \mathrm{m}$ a été démontrée

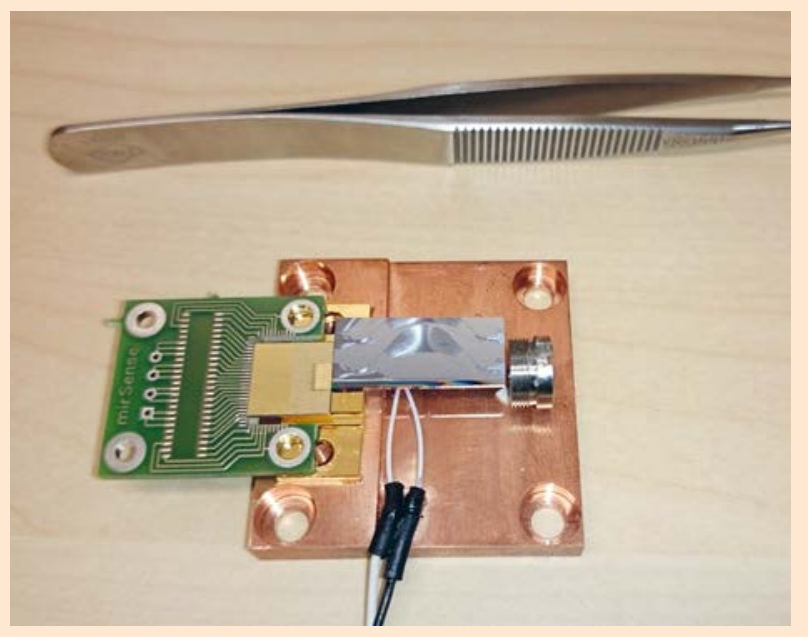

\section{Obtenir le meilleur de l'innovation, c'est notre métier.}

\section{Sensibilité élevée, temps de \\ réponse court et courant \\ d'obscurité très faible - nos \\ photodiodes à avalanche sont \\ les détecteurs optimaux pour \\ des applications LIDAR}

Figure 5. Une barrette de QCL avec un AWG (arrayed waveguide grating).

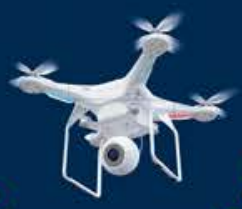




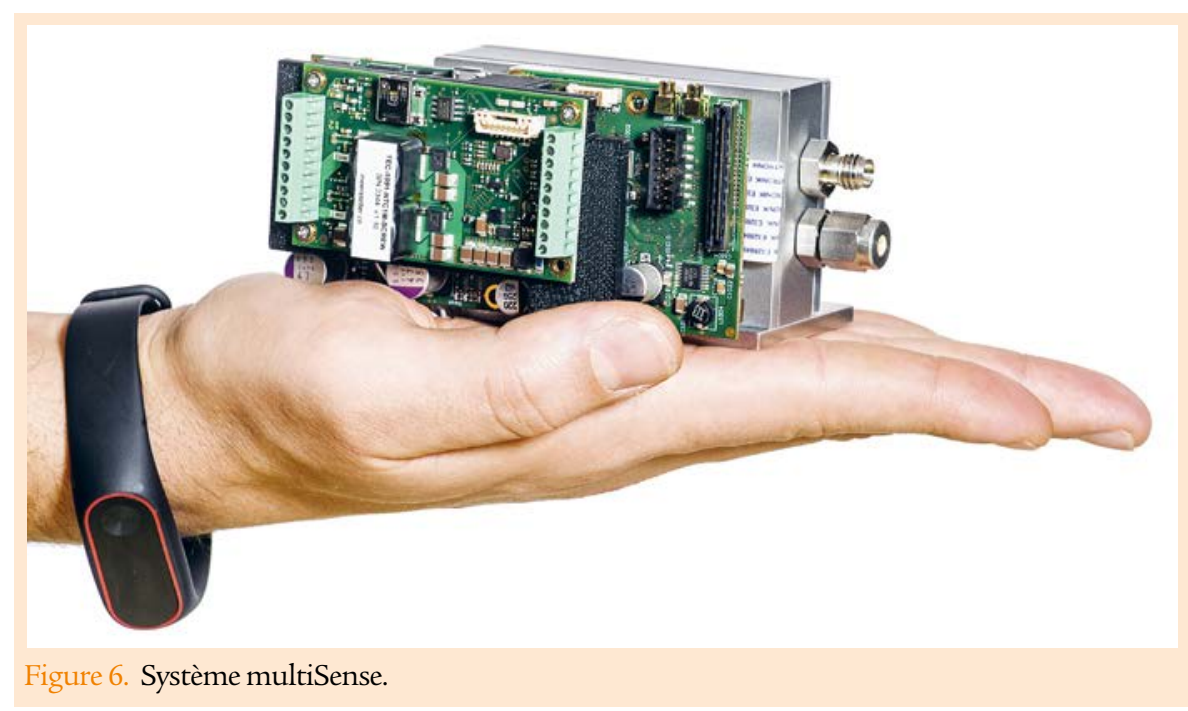

au CEA-LETI en utilisant un corps en SIGe et des couches guidantes en silicium. Des niveaux de pertes aussi bas que quelques $\mathrm{dB} / \mathrm{cm}$ ont été obtenus sur toute la plage 4-8 $\mu \mathrm{m}$ [9]. Cela a ouvert la voie à la réalisation de fonctions d'une taille de l'ordre du centimètre sans introduire de pertes prohibitives.

\section{Barrettes de QCL multiplexées}

C'est un exemple parfait du mariage des technologies QCL et silicium. La versatilité des réseaux métalliques de surface rend aussi possible la réalisation de barrettes de QCLs émettant chacun à une longueur d'onde différente, créant ainsi un peigne de longueurs d'onde. Il suffit alors d'allumer successivement chacun des lasers pour obtenir une source accordable. Les avantages de cette configuration par rapport à un système de cavité externe sont nombreux. D'une part, pour chaque longueur d'onde émise on trouve les caractéristiques d'un laser mono-fréquence, c'est-à-dire une très faible largeur de raie, une grande stabilité temporelle et une connaissance absolue de la longueur d'onde. D'autre part, on trouve une accordabilité en longueur d'onde qui peut atteindre 100 $\mathrm{cm}^{-1}$, suffisante pour de nombreuses applications de détection de molécules complexes. Le problème, si l'on s'en tient aux barrettes, est que chaque laser émet par sa facette, et donc on a des points d'émission différents pour chaque longueur d'onde. L'utilisation

\section{POUR EN SAVOIR PLUS} Linfield, A. Giles Davies, Nature Photonics, 2011, $5: 306$ 98: 181102 98: 181106 Physics Letters, 2010, $96: 161105$ Express 2014, $22: 508$ Express, 2015, 23:26168
Plusieurs PME européennes développent depuis quelques années ces systèmes. Les systèmes de spectroscopie optique actuels sont volumineux ( 20 litres, 20 kilogrammes) ce qui limite la généralisation de leur utilisation dans certains domaines d'application (portabilité, applications grand public...). Dans une démarche de miniaturisation, des équipes proposent déjà l'intégration de toutes les fonctionnalités d'un système de spectroscopie optique sur une seule puce (lab-on-chip) par intégration hétérogène de QCL avec des circuits photoniques silicium [10]. La dernière fonction qu'il est nécessaire d'intégrer est le détecteur. Il semble pour l'instant compliqué d'avoir un détecteur optique moyen infrarouge de qualité sur le silicium même si certaines équipes travaillent dessus. Une alternative intéressante est l'utilisation d'un effet photo-acoustique [11] permettant d'utiliser des microphones à la place des détecteurs optiques. Cela ouvre la voie à l'intégration de microphones MEMS, c'est-à-dire réalisés sur la filière silicium également. Les premiers produits issus de cette approche sont commercialement disponibles (figure 6 ; produit multiSense de mirSense).

Il n'est pas loin le temps où nous aurons tous un QCL à la maison !

[1] https://www.picarro.com/; https://www.northropgrumman.com/Capabilities/CIRCM

[2] J. Faist, F. Capass o, D.L. Sivco, C. Sirtori, A.L. Hutchinson, A.Y. Cho, Science, 1994, 264 : 553

[3] S. Barbieri, M. Ravaro, P. Gellie, G. Santarelli, C. Manquest, C. Sirtori, S.P. Khanna, E.H.

[4] A. Hugi, G. Villares, S. Blaser, H.C. Liu, J. Faist, Nature, 2012, 492 : 229

[5] Y. Bai, N. Bandyopadhyay, S. Tsao, S. Slivken, M. Razeghi. Applied Physics Letters, 2011,

[6] Q.Y. Lu, Y. Bai, N. Bandyopadhyay, S. Slivken, M. Razeghi, Applied Physics Letters, 2011,

[7] M. Carras, G. Maisons, B. Simozrag, M. Garcia, O. Parillaud, J. Massies, X. Marcadet, Applied

[8] R. Soref. SPIE Photonics West, paper 6898-5, Silicon Photonics III, 2008

[9] M. Brun, P. Labeye, G. Grand, J.-M. Hartmann, F. Boulila, M. Carras, Sergio Nicoletti, Optics

[10] P. Barritault, M. Brun, P. Labeye, J.-M. Hartmann, F. Boulila, M. Carras, S. Nicoletti, Optics

[11] Miklós, András, Peter Hess, Zoltán Bozóki. Review of Scientific Instruments 72(4) : 1937 (2012) 